\title{
Anti-Zeno-based dynamical control of the unfolding of quantum Darwinism
}

\author{
Salvatore Lorenzo $\odot,{ }^{1}$ Mauro Paternostro, ${ }^{2}$ and G. Massimo Palma $\oplus^{1,3}$ \\ ${ }^{1}$ Dipartimento di Fisica e Chimica-Emilio Segrè, Università degli Studi di Palermo, via Archirafi 36, I-90123 Palermo, Italy \\ ${ }^{2}$ Centre for Theoretical Atomic, Molecular, and Optical Physics, School of Mathematics and Physics, \\ Queen's University, Belfast BT7 1NN, United Kingdom \\ ${ }^{3}$ NEST, Istituto Nanoscienze-CNR, Piazza S. Silvestro 12, 56127 Pisa, Italy
}

(Received 3 August 2019; accepted 23 December 2019; published 18 February 2020)

\begin{abstract}
We combine the collisional picture for open system dynamics and the control of the rate of decoherence provided by the quantum (anti-)Zeno effect to illustrate the temporal unfolding of the redundant encoding of information into a multipartite environment that is at the basis of quantum Darwinism, and to control it. The rate at which such encoding occurs can be enhanced or suppressed by tuning the dynamical conditions of system-environment interaction in a suitable and remarkably simple manner. This would help the design of a new generation of quantum experiments addressing the elusive phenomenology of quantum Darwinism and thus its characterization.
\end{abstract}

DOI: 10.1103/PhysRevResearch.2.013164

\section{INTRODUCTION}

Quantum Darwinism (QD) is an interesting theoretical framework that strives at explaining the emergence of objective reality out of quantum superpositions, a most fundamental question in modern quantum theory, in terms of the proliferation or redundant records of the quantum state of a quantum system in the environment [1].

The basic idea is that, due to their joint interaction, an environment gets entangled with the system, thus acquiring information about its state $[2,3]$. The set of states which get entangled with the environment, the so-called pointer states, are the eigenstates of the observable of the system that enters the coupling with the environment $[4,5]$. Pointer states are left undisturbed by the mutual interaction with the environment, but their coherent superposition gets entangled with it. Any external observer who can access the environment can thus acquire information about the system [6,7].

A second important assumption of the quantum Darwinism model is that the environment is not a single block but rather a collection of independent units, each of which is endowed with accessible information about the system state, which has been redundantly encoded in the environment through the entangling process mentioned above [8-10]. Different observers having access to separate environmental fragments will have access to the same shared information about the system, which will thus become an element of objective reality, and thus an inherently classical quantity [7,11]. More formally, the assumption is that an initial coherent superposition of pointer states of the system $|\Psi\rangle_{S}=\sum_{k} \psi_{k}\left|\pi_{k}\right\rangle_{S}$ evolves into a joint

Published by the American Physical Society under the terms of the Creative Commons Attribution 4.0 International license. Further distribution of this work must maintain attribution to the author(s) and the published article's title, journal citation, and DOI. system-environment state with a branching structure

$$
\left|\Psi_{\mathrm{SE}}\right\rangle=\sum_{k} \psi_{k}\left|\pi_{k}\right\rangle_{S} \bigotimes_{j=1}^{n}\left|\eta_{k}\right\rangle_{j},
$$

where $n$ is the number of elements of the environment. Equation (1) shows that the information about the system pointer state $\left|\pi_{k}\right\rangle_{S}$ is imprinted into multiple copies of environmental states $\left|\eta_{k}\right\rangle$, thus becoming accessible to individual, distinct observers, that access separate fragments of the environment.

The emergence of quantum Darwinism has been extensively studied in recent literature, both theoretically and experimentally [12-17], and its links with other phenomena characterizing quantum and open quantum systems, such as nonMarkovianity [18-22], noncontextuality inequalities [23], and spectrum broadcasting structures [6] have been addressed and studied. Remarkably, while quantum Darwinism is implied by spectrum broadcasting, the reverse is not the case. In general, additional requests-resulting in the so-called strong quantum Darwinism [24] - have to be invoked in order to establish an equivalence with spectral broadcasting structures. Despite such suggestive result, it is still unclear when strong quantum Darwinism, and thus spectrum broadcasting, deviates from the more standard definition of Darwinism. This evidences the only partial understanding that we currently have of the Darwinistic phenomenology [7,8,11,18,24-27].

Among the points that remain to be understood is the role of quantum Darwinism in the occurrence of the quantum-toclassical transition [28], particularly in relation to the mechanism of its temporal unfolding. In this work we address this issue by adopting a collision-model approach to open quantum system dynamics [29-32] in which the interaction with the environment, consisting of an infinite number of identical elements dubbed ancillae, takes place through a sequence of rapid interactions (collisions) between the system and the ancillae. This is very close to real physical situations, such as, e.g., learning about the state of a system by observing 
the photons that are scattered by it [33], and has been recently successfully used to dig into the Darwinistic phenomenology. In this respect, our investigation moves along the lines of the works reported in Refs. [34,35], where the dynamical scattering of photons by a particle is studied from the perspective of characterizing quantum Darwinism. We show that not only does our approach provides a transparent picture of the process of successive redundant encoding of information that ultimately results in the emergence of objective reality, but also it allows for the design of simple control strategies for the harnessing of quantum Darwinism itself. We make use of the dynamical version of the quantum Zeno effect [36], which relies not on fast measurements but on cleverly arranged dynamical conditions [37]. Through this, we show that the rate at which information is spread across an environmentconsisting of many units that interact with a system by collisions - can be reduced or enhanced, and thus ultimately controlled. In turn, we believe that such (remarkably simple) control strategies can be fruitfully exploited for further experimental investigations along the lines of Refs. [12-17], and thus used to critically address the role of quantum Darwinism in the transition of quantum systems to classicality.

Our analysis shares with Ref. [27] the aim of providing a time-resolved assessment of the unfolding of objective reality. While the authors of Ref. [27] focus on quantum state discrimination and a central-system model to witness the proliferation of redundant information, our goals verge towards the demonstration of controllability of the process of information transfer that is the basis of the emergence of quantum Darwinism.

The remainder of this paper is structured as follows. In Sec. II we introduce our collision model of pure decoherence and derive the unfolding of quantum Darwinism. Section III is dedicated to the description of our approach to the control of such unfolding: we show that the judicious arrangement of a quantum Zeno mechanism is effective in inhibiting the spreading of redundant information from the system to fractions of the environment (cf. Sec. III A), while an anti-Zeno-like effect accelerates such effect towards a faster onset of quantum Darwinism (cf. Sec. III B). Finally, in Sec. IV we draw the conclusions that can be gathered from our work and briefly address the avenues that they open.

\section{UNFOLDING OF DARWINISM IN A MEMORYLESS COLLISION MODEL OF DECOHERENCE}

In order to illustrate how quantum Darwinism unfolds in time we address decoherence resulting from a simple memoryless collision model. While providing an intuitive mechanism for quantum Darwinism, the collisional picture is rich enough to allow for the grasping of the subtleties associated to the emergence of objective reality.

As in a standard collisional model of open system dynamics, the environment consists of a large ensemble of subenvironments, dubbed ancillae. The irreversible reduced dynamics of the system is described in terms of a sequence of brief interactions (collisions) between the system and the ancillae. In the memoryless scenario the number of ancillae in the environment is infinite and the system never collides twice with the same ancilla [38-40]. If we assume $n \gg 1$ and the ancillae

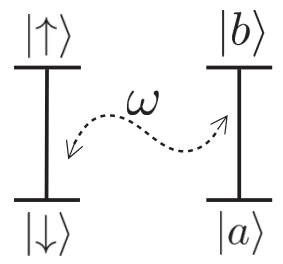

(a)

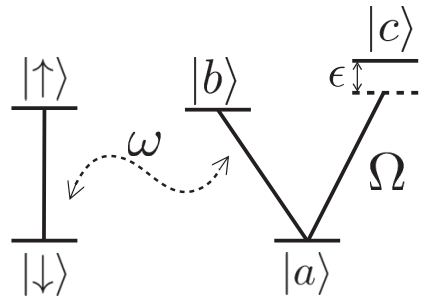

(b)
FIG. 1. Schematic representations of the interaction models for the temporal unfolding of quantum Darwinism considered in this work. Panel (a) illustrates the configuration of system-environment interactions that give rise to redundant encoding of information about the system onto the state of the environmental elements via repeated collisions. Each involves subenvironments embodied by two-level systems interacting with $\mathcal{S}$ according to the Hamiltonian in Eq. (2). Panel (b) is for the depletion (enhancement) of the rate of redundant encoding achieved through a (anti-)Zeno-like effect. We induce this by choosing three-level systems as ancillae. While the $|a\rangle \leftrightarrow|c\rangle$ is driven with a (dimensionless) Rabi frequency $\Omega$, the $|a\rangle \leftrightarrow|b\rangle$ one is directly affected by the collision with the system. Different regimes of redundant encoding are achieved by adjusting the detuning $\epsilon$.

all prepared in the same state $\hat{\eta}$, after $\ell$ collisions the joint system-environment state will be $\hat{\rho}_{\mathcal{S R}}=\hat{U}_{\{\ell\}}\left(\hat{\rho}_{0} \otimes \hat{\eta}^{\otimes n}\right) \hat{U}_{\{\ell\}}^{\dagger}$ where $\hat{U}_{\{\ell\}}=\hat{U}_{\ell} \hat{U}_{\ell-1} \cdots \hat{U}_{1}$ and $\hat{U}_{k}$ describes the collision between the $k$ th ancilla and the system. To be specific, let us now consider a two-level system $\mathcal{S}$ and an environment $\mathcal{R}$, in turn composed by a large collection of two-level systems $\mathcal{R}_{n}$. The $\mathcal{S}-\mathcal{R}$ collisions are generated by the following interaction Hamiltonian [cf. Fig. 1(a)]:

$$
\hat{H}_{n}=\hbar \omega \hat{\sigma}_{\mathcal{S}}^{z} \otimes \hat{\sigma}_{\mathcal{R}_{n}}^{x},
$$

where $\hat{\sigma}_{\mathcal{S}}^{z}=|\uparrow\rangle\left\langle\left.\uparrow\right|_{\mathcal{S}}-\mid \downarrow\right\rangle\left\langle\left.\downarrow\right|_{\mathcal{S}}\right.$ and $\left.\hat{\sigma}_{\mathcal{R}_{j}}^{x}=\mid a\right\rangle\left\langle\left. b\right|_{\mathcal{R}_{j}}+\mid b\right\rangle\left\langle\left. a\right|_{\mathcal{R}_{j}}\right.$ are the $z$ - and $x$-Pauli matrices for $\mathcal{S}$ and $\mathcal{R}_{j}$, respectively. In order to fix the ideas and with no loss of generality we ignore at this stage any free system or ancilla dynamics. The collisions are thus described by a rotation operator on the ancilla conditioned on the state of the system

$$
\hat{U}=\exp \left\{-i \omega \hat{\sigma}_{\mathcal{S}}^{z} \otimes \hat{\sigma}_{\mathcal{R}_{n}}^{x} \tau\right\},
$$

where $\tau$ stands for the collision time, i.e., the duration of each collision. Such dynamics will induce entanglement between the system and an increasing number of ancillae as the collisions take place. Starting from $\Psi_{0}=(\alpha|\downarrow\rangle+\beta|\uparrow\rangle) \bigotimes_{j=1}^{n}|a\rangle$ we can write the $\mathcal{S}$ - $\mathcal{R}$ joint state after $\ell$ collisions as

$$
\left|\Psi_{\ell}\right\rangle=\left(\alpha|\downarrow\rangle \bigotimes_{j=1}^{\ell}\left|\varphi^{+}\right\rangle_{\mathcal{R}_{j}}+\beta|\uparrow\rangle \bigotimes_{j=1}^{\ell}\left|\varphi^{-}\right\rangle_{\mathcal{R}_{j}}\right) \bigotimes_{j=\ell+1}^{n}|a\rangle_{\mathcal{R}_{j}},
$$

where $\left|\varphi^{ \pm}\right\rangle=\cos (\omega \tau)|a\rangle \pm i \sin (\omega \tau)|b\rangle$. The corresponding reduced density matrix $\rho_{\mathcal{S}}^{\ell}$ is

$$
\rho_{\mathcal{S}}^{\ell}=\operatorname{tr}_{\mathcal{R}}\left\{\left|\Psi_{\ell}\right\rangle\left\langle\Psi_{\ell}\right|\right\}=\left(\begin{array}{cc}
|\alpha|^{2} & \alpha \beta^{*} \kappa^{\ell} \\
\alpha^{*} \beta \kappa^{* \ell} & |\beta|^{2}
\end{array}\right)
$$

with $\kappa=\left|\left\langle\varphi^{-}\right\rangle \varphi^{+}\right|=|\cos (2 \omega \tau)|$. The above expressions show clearly that the states $|\downarrow\rangle_{\mathcal{S}}$ and $|\uparrow\rangle_{\mathcal{S}}$ are pointer states 
and the ancillae, collision after collision, gradually acquire information about the system.

Typically many collisions, i.e., many subenvironments, are necessary to distinguish pointer states. The number of times that this information can be independently extracted is taken as a measure of objectivity. The mutual information between the system and a subset $m$ of ancillas after $\ell$ collisions

$$
\mathcal{I}\left(\mathcal{S}, \mathcal{F}_{m}^{\ell}\right)=S\left(\rho_{\mathcal{S}}^{\ell}\right)+S\left(\rho_{\mathcal{F}_{m}}^{\ell}\right)-S\left(\rho_{\mathcal{S} \mathcal{F}_{m}}^{\ell}\right)
$$

is the information about $\mathcal{S}$ available from $\mathcal{F}_{m}$. The emergence of Darwinistic behavior can be detected by the existence of a broad horizontal plateau in the plot of $\mathcal{I}\left(\mathcal{S}, \mathcal{F}_{m}^{\ell}\right)$ as a function of the fragment dimension $m$. When $\mathcal{I}\left(\mathcal{S}, \mathcal{F}_{m}^{\ell}\right)$ quickly raise to $S\left(\rho_{\mathcal{S}}^{\ell}\right)$, almost all information accessible on the system is encoded in a small fraction of the environment. We call $\mathcal{F}_{\delta}^{\ell}$ the smallest fraction of the environment such that, for any arbitrarily picked information gap $\delta$, we achieve

$$
\mathcal{I}\left(\mathcal{S}, \mathcal{F}_{\delta}^{\ell}\right) \geqslant(1-\delta) S\left(\rho_{\mathcal{S}}^{\ell}\right) .
$$

We can thus define the redundancy as

$$
R=\ell / \mathcal{F}_{\delta}^{\ell},
$$

which provides a quantitative estimate of the number of distinct subenvironments that supply classical information about $\mathcal{S}$, up to the deficit $\delta$.

The off-diagonal matrix elements of $\rho_{\mathcal{S}}^{\ell}$ decay as $\kappa^{\ell}=$ $e^{-\Gamma \ell}$ where we have defined the decay constant $\Gamma=-\ln \kappa$. After $\tilde{\ell}=\Gamma^{-1}$ collisions, $\rho_{\mathcal{S}}^{\tilde{\ell}}$ will be fully decohered. Further collisions will thus take place with the system that is in a state with no quantum coherence. This implies that the size of environmental fragments which have nearly complete knowledge about the state of the system is $\mathcal{O}\left(\tilde{\ell}^{-1}\right)$ and, after $n$ collisions, we expect a redundancy $R \sim-n \ln \kappa$. We will show shortly the correctness of this estimate.

Before proceeding to the analytic evaluation of the quantum mutual information, let us point out that, in the limit of very short collision time that justifies a limit to continuous time, the above dynamics can be effectively described by the dephasing master equation

$$
\frac{d \rho}{d t}=\gamma\left(\hat{\sigma}^{z} \rho \hat{\sigma}^{z}-\rho\right)
$$

with $\gamma=\omega^{2} \tau$ the decoherence rate. To evaluate the mutual information between the system and growing environmental fragments, we make extensive use of the fact that the overall system-environment state remains pure after an arbitrary number of collisions. The von Neumann entropy of $\mathcal{S}$ is easily calculated at each step as $S\left(\rho_{\mathcal{S}}^{\ell}\right)=-\sum_{j= \pm} \lambda_{j} \log _{2} \lambda_{j}$ with $\lambda_{ \pm}=\left(1 \pm \sqrt{1-4\left(1-|\kappa|^{2 \ell}\right)|\alpha|^{2}|\beta|^{2}}\right) / 2$. We now need the quantum von Neumann entropies of $\rho_{\mathcal{F}_{m}}$ and $\rho_{\mathcal{S F}}$. Let us note that, after $\ell>m$ collisions, i.e., when the $\ell$ collision involves an ancilla out from the fragment $\mathcal{F}_{m}$, the reduced density matrix of the fragment $\mathcal{F}_{m}$ is given by

$$
\rho_{\mathcal{F}_{m}}^{\ell>m}=|\alpha|^{2} \bigotimes_{j=1}^{m}\left|\varphi^{+}\right\rangle\left\langle\left.\varphi^{+}\right|_{\mathcal{R}_{j}}+|\beta|^{2} \bigotimes_{j=1}^{m} \mid \varphi^{-}\right\rangle\left\langle\left.\varphi^{-}\right|_{\mathcal{R}_{j}} .\right.
$$

This means that after the first $m$ collisions the reduced density matrix of $\mathcal{F}_{m}$ does not change further and, recalling that within (a)

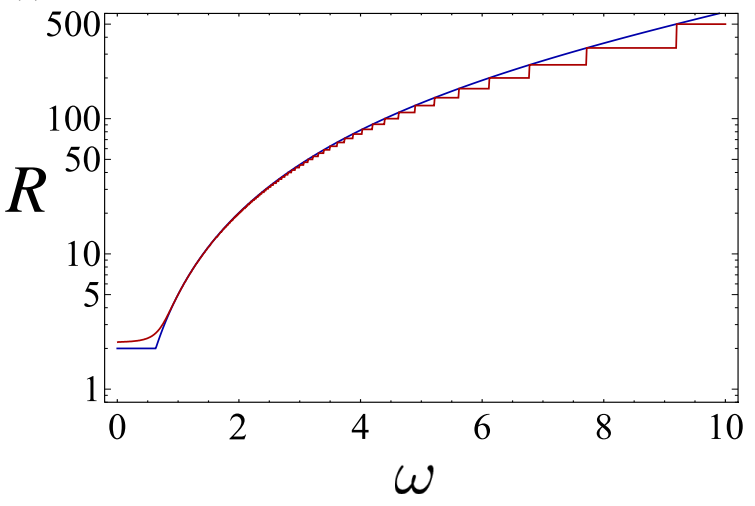

(b)

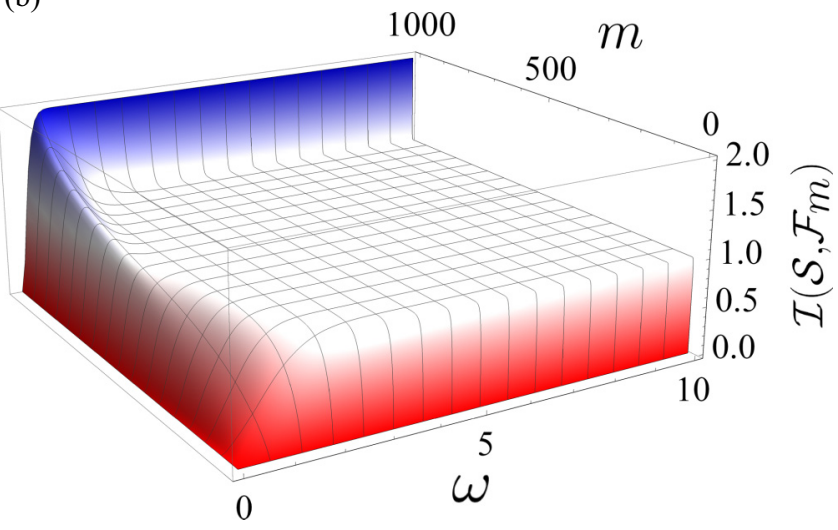

FIG. 2. (a) The red jagged line shows the redundancy $R$ versus $\omega$ after $n=10^{3}$ collisions and for $\tau=0.05$ and $\delta=0.05$. The blue curve shows the behavior of the estimated scaling law $-n \ln \kappa$. (b) We plot the mutual information $\mathcal{I}\left(\rho_{\mathcal{S}}, \mathcal{F}_{m}\right)$ versus $\omega$ and the size of the environmental fraction $m$ for the same parameters as in panel (a).

the first $m$ collisions the joint $\mathcal{S}-\mathcal{F}_{m}$ state is pure, we have that

$$
S\left(\rho_{\mathcal{F}_{m}^{\ell>m}}^{\ell m}\right)=S\left(\rho_{\mathcal{S}}^{m}\right)
$$

With similar considerations, the entropy of $\rho_{\mathcal{S} \mathcal{F}_{m}}$ turns out to be $S\left(\rho_{\mathcal{S} \mathcal{F}_{m}}^{\ell>m}\right)=S\left(\rho_{\mathcal{S}}^{\ell-m}\right)$. On the other hand, if $\ell<m$ we have that $S\left(\rho_{\mathcal{F}_{m}}^{\ell<m}\right)=S\left(\rho_{\mathcal{S}}^{\ell<m}\right)$, and $S\left(\rho_{\mathcal{S} \mathcal{F}_{m}}^{\ell<m}\right)=0$. Putting together all the above relations, the mutual information between $\mathcal{S}$ and $\mathcal{F}_{m}$ is

$$
\mathcal{I}\left(\mathcal{S}, \mathcal{F}_{m}^{\ell}\right)= \begin{cases}S\left(\rho_{\mathcal{S}}^{\ell}\right)+S\left(\rho_{\mathcal{S}}^{m}\right)-S\left(\rho_{\mathcal{S}}^{\ell-m}\right) & \text { for } m<\ell \\ 2 S\left(\rho_{\mathcal{S}}^{\ell}\right) & \text { for } m>\ell\end{cases}
$$

In Fig. 2 the redundancy $R$ is shown as a function of $\omega$ and its value is compared with $\Gamma(\tau)=-n \ln \kappa(\tau)$. The agreement between the two quantities is excellent, confirming our estimate of the redundancy in terms of the decay rate $\Gamma$. Note that $R$ is a monotonically growing function of $\omega$ as long as $2 \omega \tau \leqslant \pi / 2$. 


\section{DYNAMICAL CONTROL OF THE TEMPORAL UNFOLDING OF QUANTUM DARWINISM}

The study illustrated in the previous section shows that a collisional model is able to faithfully capture the features linked to the mechanism of temporal unfolding of quantum Darwinism, and thus account for the building up of redundant information encoding in the state of a multielement environment.

We shall now take advantage of such understanding and show that, by means of carefully designed (anti-)Zeno-like mechanisms, it is possible to control the spreading of informational redundancy, thus harnessing the unfolding discussed above. Remarkably, the control extends from dynamical inhibition all the way to the enhancement of the redundancy spreading, which we achieve by engineering the effective dephasing rate of the system. This demonstrates the significant control that can be operated on the fundamental features of quantum Darwinism, and paves the way to its use for the harnessing of the quantum-to-classical transition.

\section{A. Inhibiting the spread of redundancy via the quantum Zeno effect}

We start by addressing the controlled inhibition of quantum Darwinism. From the discussion in Sec. II it is clear that the workhorse for the achievement of this goal is control of the information that each ancilla acquires about the system. In turn, this implies the engineering of rate $\Gamma$. A way of doing this is by engineering a Zeno-like dynamics for the ancillae, which effectively freezes their dynamics during the collisional process.

The quantum Zeno effect, first pointed out by Misra and Sudarshan in their seminal work [36], simply states that the continuous observation of a system freezes its evolution. The original argument goes along the following lines: let $|\psi\rangle$ be the initial state of a system evolving according to the Hamiltonian $\hbar \hat{V}$. After a time $t$, the survival probability of the system in its initial state is $|\langle\psi|\exp \{-i \hat{V} t\}| \psi\rangle|^{2} \simeq 1-$ $\left\langle\left(\Delta^{2} \hat{V}\right)\right\rangle t^{2}+\mathcal{O}\left(t^{4}\right)$ with $\Delta^{2} \hat{V}=\left\langle\hat{V}^{2}\right\rangle-\langle\hat{V}\rangle^{2}$. Let us assume during time $t$ the system is measured $p$ times: in this case the survival probability becomes $\left[1-\left\langle\Delta^{2} \hat{V}\right\rangle(t / p)^{2}\right]^{p} \approx 1$ when $p \rightarrow \infty$. While we do not aim at discussing the considerable body of literature on the Zeno effect produced in the last three decades and spurred by Ref. [36], here we focus on an early model of constant monitoring put forward by Peres [37] to freeze the ancilla dynamics.

Let us assume that the ancillae are no longer two-level systems and instead enlarge the Hilbert space of each $\mathcal{R}_{n}$ by introducing a third state $|c\rangle$ coupled only to $|a\rangle$ as shown in Fig. 1(b). This allows us to introduce a dynamics of the subenvironment that is independent of the collisional mechanism (we dub it free), and which we use as our control tool. We thus model each dynamical step through the Hamiltonian

$$
\hat{H}_{n}=\omega \hat{\sigma}_{\mathcal{S}}^{z} \otimes e^{i \hat{h}_{n} \tau}\left(|a\rangle\left\langle\left. b\right|_{\mathcal{R}_{n}}+\mid b\right\rangle\left\langle\left. a\right|_{\mathcal{R}_{n}}\right) e^{-i \hat{h}_{n} \tau}\right.
$$

with $\hat{h}_{n}=(\Omega / \tau)\left(|a\rangle\left\langle\left. c\right|_{\mathcal{R}_{n}}+\mid c\right\rangle\left\langle\left. a\right|_{\mathcal{R}_{n}}\right)\right.$ the free Hamiltonian for the $|a\rangle \leftrightarrow|c\rangle$ transitions and $\Omega$ a dimensionless Rabi rate (we remind one that the evolution time $\tau$ due to collisions is set in our framework, and thus a constant). Eq.uation (13) is thus the interaction Hamiltonian between the system and the subenvironments written in a reference frame set by the free Hamiltonian $\hat{h}_{n}$. In what follows we will require $\Omega /(\omega \tau) \gg 1$ so as to freeze the dynamics of the subenvironments.

In the basis of $\hat{\sigma}_{\mathcal{S}}^{z}$ the corresponding step evolution operator can be written as

$$
\hat{U}=\left(\begin{array}{cc}
e^{-i \omega \tau \hat{M}} & \mathbb{O} \\
\mathbb{O} & e^{i \omega \tau \hat{M}}
\end{array}\right),
$$

where $\mathbb{O}$ is the null matrix and, in the basis $\{|c\rangle,|b\rangle,|a\rangle\}_{\mathcal{R}_{n}}$, we have

$$
M=\left(\begin{array}{ccc}
0 & -i \sin \Omega & 0 \\
i \sin \Omega & 0 & \cos \Omega \\
0 & \cos \Omega & 0
\end{array}\right) .
$$

For $\Omega \in[0, \pi / 2], M$ has eigenvalues $\lambda_{ \pm}= \pm 1$ and $\lambda_{0}=0$. The associated eigenvectors are

$$
\begin{aligned}
\left|\varphi^{ \pm}\right\rangle & =\frac{1}{\sqrt{2}}(\cos \Omega|a\rangle \pm|b\rangle-i \sin \Omega|c\rangle), \\
\left|\varphi^{0}\right\rangle & =(\sin \Omega|a\rangle+i \cos \Omega|c\rangle) .
\end{aligned}
$$

The reduced density matrix of the system is given again by Eq. (5). However, the decoherence factor is modified
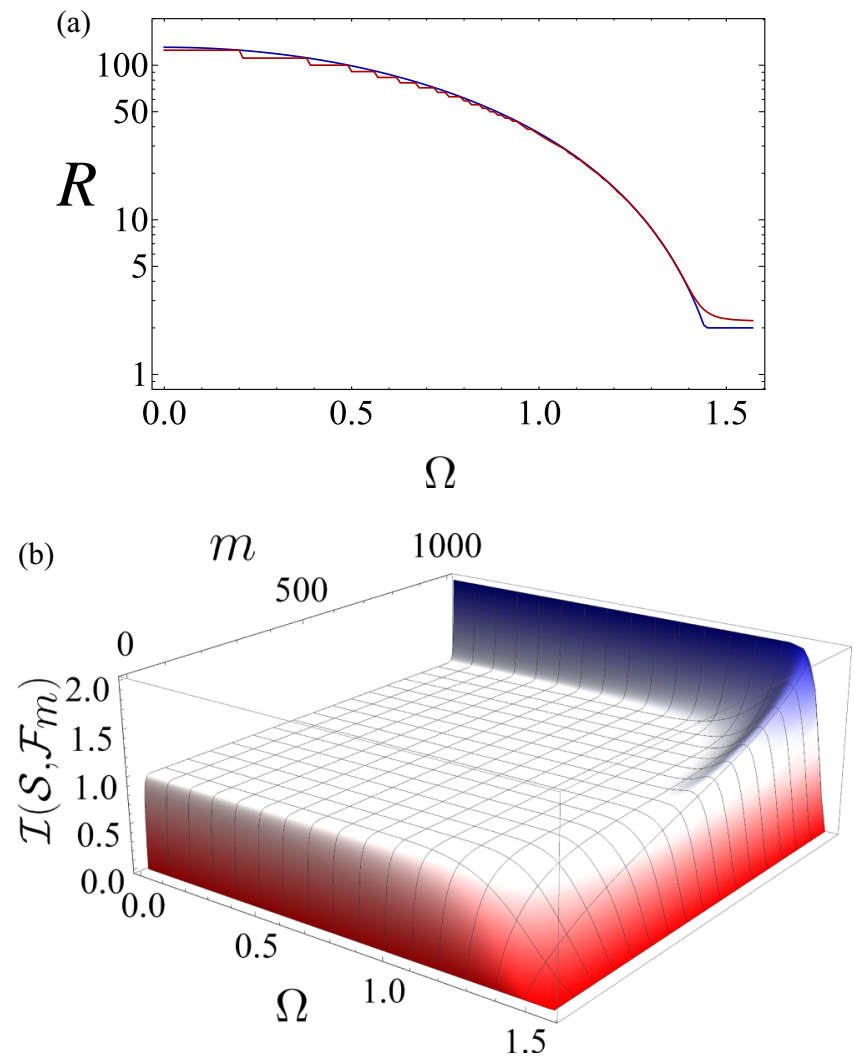

FIG. 3. (a) We plot the redundancy $R$ (red jagged line) against the dimensionless quantity $\Omega \in[0, \pi / 2]$ for $n=10^{3}$ collisions, each lasting a time $\tau=0.05$, for $\omega=5$ and $\delta=0.05$. For $\Omega>\omega \tau$, the redundancy is reduced. The blue curve shows the behavior of the esitmate $-n \log (\kappa)$. (b) We show the mutual information $\mathcal{I}\left(\rho_{\mathcal{S}}, \mathcal{F}_{m}\right)$ versus $\Omega$ and the fraction $m$ of subenvironments considered. 


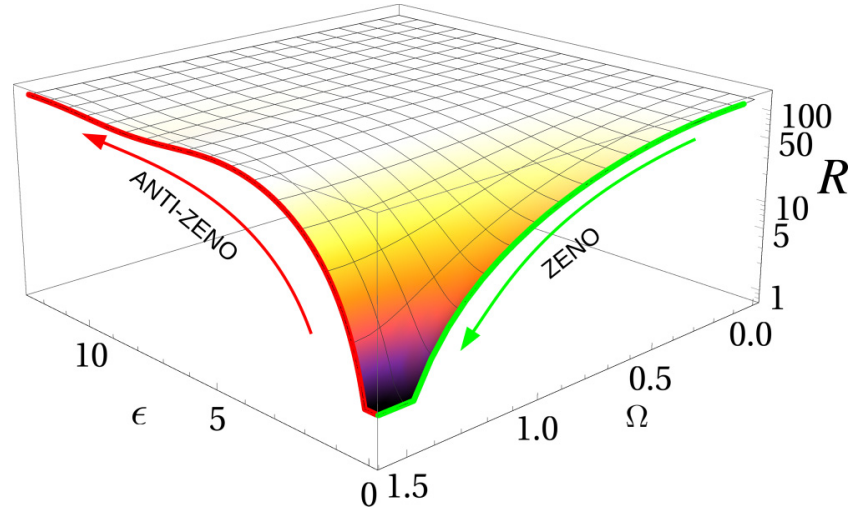

FIG. 4. Redundancy $R_{\delta}$ after $N=10^{3}$ collisions, $\tau=0.05$ and $\omega=5$ versus $\Omega$ and $\epsilon$. The green curve is the same as in Fig. 3: starting from $\Omega \sim 1.5$ and increasing the detuning, the redundancy increases (red curve).

dynamically as

$\kappa=\sum_{j= \pm, 0} e^{-2 i \omega \tau \lambda_{j}}\left|\left\langle\varphi^{j} \mid a\right\rangle\right|^{2}=\cos (2 \omega \tau) \cos ^{2} \Omega+\sin ^{2} \Omega$,

which corresponds to a new dephasing rate [cf. Eq. (9)] reading $\gamma=\omega^{2} \tau \cos ^{2} \Omega$. The behavior of the redundancy $R$ associated with such a modified dynamics is shown in Fig. 3, which demonstrates that large values of $\Omega$-at set values of $\omega \tau$-correspond to a depleted information spreading effect: The fact that free Rabi flopping in the $\{|a\rangle,|c\rangle\}_{\mathcal{R}_{n}}$ subspace induced by a large value of $\Omega$ freeze the evolution of the subenvironments by projecting their state onto $|a\rangle_{\mathcal{R}_{n}}$ at a rate that exceeds the effects of any collision.

\section{B. Enhancing the spreading of redundancy via the quantum anti-Zeno effect}

We now reverse our goal and look for a strategy that allows for the enhancement of the information spreading effect that unfolds quantum Darwinism. Taking advantage of the analysis presented in Sec. III A, we aim at modeling an effective anti-Zeno mechanism that accelerates the rate of collisional spin flip induced by the $\mathcal{S}-\mathcal{R}_{n}$ interaction. The solution is remarkably simple.

We model each step again as in Eq. (13). However, we now consider the possibility to have an energy detuning $2 \epsilon$ between $|a\rangle$ and $|c\rangle$, which changes the free Hamiltonian $\hat{h}_{n}$ as

$$
h_{n}=-2(\epsilon / \tau)|c\rangle\left\langle\left. c\right|_{\mathcal{R}_{n}}+(\Omega / \tau)\left(|a\rangle\left\langle\left. c\right|_{\mathcal{R}_{n}}+\mid c\right\rangle\left\langle\left. a\right|_{\mathcal{R}_{n}}\right)\right.\right.
$$

and, in turn, matrix $M$ as

$$
M=\left(\begin{array}{ccc}
0 & \frac{i \Omega e^{-i \epsilon} \sin v}{v} & 0 \\
-\frac{i \Omega e^{i \epsilon} \sin v}{v} & 0 & e^{i \epsilon}\left(\cos v-\frac{i \epsilon \sin v}{v}\right) \\
0 & e^{-i \epsilon}\left(\cos v+\frac{i \epsilon \sin v}{v}\right) & 0
\end{array}\right),
$$

where $v=\sqrt{\epsilon^{2}+\Omega^{2}}$. Following steps akin to those presented in Sec. III A, it is straightforward to evaluate the affected dephasing rate and the new redundancy $R$, which now depends on

$$
\kappa=\frac{\Omega^{2} \sin ^{2} v+\cos (2 \tau \omega)\left(v^{2} \cos ^{2} v+\epsilon^{2} \sin ^{2} v\right)}{v^{2}} .
$$

This expression goes back to Eq. (17) for $\epsilon \rightarrow 0$ and, on the other hand, when $\epsilon \gg \Omega$ the state $|c\rangle$ results practically decoupled from $|a\rangle$ leading to a redundancy that can be larger than the one associated with small detuning and large values of coupling $\Omega$, as shown in Fig. 4, thus demonstrating the possibility to enhance the information spreading.

\section{CONCLUSIONS}

By making use of a collisional approach to open system dynamics, we have shed light on the temporal unfolding of the redundant encoding of information that is at the basis of the emergence of quantum Darwinism. Remarkably, this has opened the way to the design of quantum Zeno-based techniques for the control of the information spreading rate, which can be enhanced or depleted by engineering systemenvironment couplings and suitably arranging the spectrum of the subenvironments. We believe this work draws a pathway to the design of experimental settings able to mimic and thus validate the features and controlled dynamics discussed here. In turn, this will contribute further to the clarification of the role that quantum Darwinism plays in the understanding of the quantum-to-classical transition [17].

\section{ACKNOWLEDGMENTS}

We are grateful to W. H. Zurek for discussions. We acknowledge support under PRIN project 2017SRNBRK QUSHIP funded by MIUR, the EU Collaborative project TEQ (Grant Agreement No. 766900), the DfE-SFI Investigator Programme (Grant No. 15/IA/2864), COST Action CA15220, the Royal Society Wolfson Research Fellowship (RSWF\R3\183013), and the Leverhulme Trust Research Project Grant (Grant No. RGP-2018-266). G.M.P. would like to thank the Kavli Institute for Theoretical Physics, UC Santa Barbara, for its hospitality supported in part by the National Science Foundation under Grant No. NSF PHY-1748958.
[1] W. H. Zurek, Quantum darwinism, Nat. Phys. 5, 181 (2009).

[2] W. H. Zurek, Decoherence, einselection, and the quantum origins of the classical, Rev. Mod. Phys. 75, 715 (2003).

[3] R. Blume-Kohout and W. H. Zurek, A simple example of "Quantum Darwinism": Redundant information storage in many-spin environments, Found. Phys. 35, 1857 (2005).

[4] W. H. Zurek, Pointer basis of quantum apparatus: Into what mixture does the wave packet collapse? Phys. Rev. D 24, 1516 (1981). 
[5] W. H. Zurek, Environment-induced superselection rules, Phys. Rev. D 26, 1862 (1982).

[6] J. K. Korbicz, P. Horodecki, and R. Horodecki, Objectivity in a Noisy Photonic Environment through Quantum State Information Broadcasting, Phys. Rev. Lett. 112, 120402 (2014).

[7] R. Horodecki, J. K. Korbicz, and P. Horodecki, Quantum origins of objectivity, Phys. Rev. A 91, 032122 (2015).

[8] M. Zwolak, H. T. Quan, and W. H. Zurek, Redundant imprinting of information in nonideal environments: Objective reality via a noisy channel, Phys. Rev. A 81, 062110 (2010).

[9] M. Zwolak and W. H. Zurek, Redundancy of einselected information in quantum Darwinism: The irrelevance of irrelevant environment bits, Phys. Rev. A 95, 030101(R) (2017).

[10] M. Zwolak, C. J. Riedel, and W. H. Zurek, Amplification, decoherence, and the acquisition of information by spin environments, Sci. Rep. 6, 25277 (2016).

[11] T. P. Le and A. Olaya-Castro, Objectivity (or lack thereof): Comparison between predictions of quantum Darwinism and spectrum broadcast structure, Phys. Rev. A 98, 032103 (2018).

[12] M. A. Ciampini, G. Pinna, P. Mataloni, and M. Paternostro, Experimental signature of quantum Darwinism in photonic cluster states, Phys. Rev. A 98, 020101(R) (2018).

[13] A. M. Burke, R. Akis, T. E. Day, G. Speyer, D. K. Ferry, and B. R. Bennett, Periodic Scarred States in Open Quantum Dots as Evidence of Quantum Darwinism, Phys. Rev. Lett. 104, 176801 (2010).

[14] R. Brunner, R. Akis, D. K. Ferry, F. Kuchar, and R. Meisels, Coupling-Induced Bipartite Pointer States in Arrays of Electron Billiards: Quantum Darwinism in Action? Phys. Rev. Lett. 101, 024102 (2008).

[15] M.-C. Chen, H.-S. Zhong, Y. Li, D. Wu, X.-L. Wang, L. Li, N.-L. Liu, C.-Y. Lu, and J.-W. Pan, Emergence of classical objectivity of quantum darwinism in a photonic quantum simulator, Sci. Bull. 64, 580 (2019).

[16] T. K. Unden, D. Louzon, M. Zwolak, W. H. Zurek, and F. Jelezko, Revealing the Emergence of Classicality Using Nitrogen-Vacancy Centers, Phys. Rev. Lett. 123, 140402 (2019).

[17] P. Ball, Quantum Darwinism, an idea to explain objective reality, passes first tests, Quanta Magazine, Simons Foundation, 22 July 2019, https://www.quantamagazine.org/quantumdarwinism-an-idea-to-explain-objective-reality-passes-firsttests-20190722/.

[18] A. Lampo, J. Tuziemski, M. Lewenstein, and J. K. Korbicz, Objectivity in the non-Markovian spin-boson model, Phys. Rev. A 96, 012120 (2017).

[19] G. Pleasance and B. M. Garraway, Application of quantum Darwinism to a structured environment, Phys. Rev. A 96, 062105 (2017).

[20] G. L. Giorgi, F. Galve, and R. Zambrini, Quantum darwinism and non-markovian dissipative dynamics from quantum phases of the spin-1/2 XX model, Phys. Rev. A 92, 022105 (2015).
[21] F. Galve, R. Zambrini, and S. Maniscalco, Non-Markovianity hinders quantum Darwinism, Sci. Rep. 6, 19607 (2016).

[22] N. Milazzo, S. Lorenzo, M. Paternostro, and G. M. Palma, Role of information backflow in the emergence of quantum darwinism, Phys. Rev. A 100, 012101 (2019).

[23] R. D. Baldijao and M. Terra Cunha, Classical limits and contextuality in a scenario of multiple observers, arXiv:1811.00615.

[24] T. P. Le and A. Olaya-Castro, Strong Quantum Darwinism and Strong Independence are Equivalent to Spectrum Broadcast Structure, Phys. Rev. Lett. 122, 010403 (2019).

[25] M. Zwolak, H. T. Quan, and W. H. Zurek, Quantum Darwinism in a Mixed Environment, Phys. Rev. Lett. 103, 110402 (2009).

[26] P. Mironowicz, P. Należyty, P. Horodecki, and J. K. Korbicz, System information propagation for composite structures, Phys. Rev. A 98, 022124 (2018).

[27] P. Mironowicz, J. K. Korbicz, and P. Horodecki, Monitoring of the Process of System Information Broadcasting in Time, Phys. Rev. Lett. 118, 150501 (2017).

[28] S. Campbell, B. Çakmak, Ö. E. Müstecaplığlu, M. Paternostro, and B. Vacchini, Collisional unfolding of quantum Darwinism, Phys. Rev. A 99, 042103 (2019).

[29] M. Ziman, P. Štelmachovič, V. Bužek, M. Hillery, V. Scarani, and N. Gisin, Diluting quantum information: An analysis of information transfer in system-reservoir interactions, Phys. Rev. A 65, 042105 (2002).

[30] M. Ziman and V. Bužek, All (qubit) decoherences: Complete characterization and physical implementation, Phys. Rev. A 72, 022110 (2005).

[31] M. Ziman, P. Štelmachovič, and V. Bužek, Description of quantum dynamics of open systems based on collision-like models, Open Syst. Inf. Dyn. 12, 81 (2005).

[32] M. Ziman and V. Bužek, Open system dynamics of simple collision models, Quant. Dyn. Inf. 199 (2010).

[33] H. Ollivier, D. Poulin, and W. H. Zurek, Objective Properties from Subjective Quantum States: Environment as a Witness, Phys. Rev. Lett. 93, 220401 (2004).

[34] C. J. Riedel and W. H. Zurek, Quantum Darwinism in an Everyday Environment: Huge Redundancy in Scattered Photons, Phys. Rev. Lett. 105, 020404 (2010).

[35] C. J. Riedel and W. H. Zurek, Redundant information from thermal illumination: quantum Darwinism in scattered photons, New J. Phys. 13, 073038 (2011).

[36] B. Misra and E. C. G. Sudarshan, The Zeno's paradox in quantum theory, J. Math. Phys. 18, 756 (1977).

[37] A. Peres, Zeno paradox in quantum theory, Am. J. Phys. 48, 931 (1980).

[38] F. Ciccarello, G. M. Palma, and V. Giovannetti, Collisionmodel-based approach to non-Markovian quantum dynamics, Phys. Rev. A 87, 040103(R) (2013).

[39] F. Ciccarello, Collision models in quantum optics, Quantum Meas. Quantum Metrol. 4, 53 (2017).

[40] S. Lorenzo, F. Ciccarello, and G. M. Palma, Composite quantum collision models, Phys. Rev. A 96, 032107 (2017). 